\title{
A new model of patient-reported outcome monitoring with a clinical feedback system in ostomy care: rationale, description and evaluation protocol
}

Kirsten Lerum Indreb $\emptyset^{1 *}$ (D) Anny Aasprang ${ }^{2}$, Torill Elin Olsen ${ }^{3}$ and John Roger Andersen ${ }^{4,2}$

\begin{abstract}
Background: Living with an ostomy can be challenging and adapting to life with an ostomy can be particularly complex, with regard to both the physical and psychosocial aspects. Follow-up with a stoma care nurse (SCN) is usually performed after surgery to support the adaptation process. In the present paper, we describe a new model of ostomy care, where a clinical feedback system (CFS) is implemented in order to improve the adaption process of patients with an ostomy. We also present a plan for evaluating patients experience with the CFS and their clinical outcomes.
\end{abstract}

Methods: In this study, we include patients who had recently performed colostomy, ileostomy, or urostomy surgery. The intervention includes self-reported measures for adaptation to life with an ostomy and healthrelated quality of life (HRQoL), as well as patient experiences and satisfaction recorded by the clinical feedback system. The measures are electronically assessed before each clinical consultation at 3, 6, and 12 months after surgery. The scores are instantly analysed and graphically presented for use during the consultation and the patient and the SCN can discuss the findings. Patient experiences and satisfaction with care will be measured with the Generic Short Patient Experiences Questionnaire. Adaptation to the life with ostomy will be measured with the Ostomy Adjustment Scale, and HRQoL with the Short Form 36.

Discussion: This study presents a novel approach that could lead to improved consultation, more patient involvement, and better adaptation to life with an ostomy.

Trial register: ClinicalTrials.gov Registration Number: NCT03841071.

Date 18. February 2019 retrospectively registered.

Keywords: Ostomy, Adjustment, Adaptation, Clinical feedback system, Routine outcome measure

\footnotetext{
* Correspondence: kirsten.lerum.indrebo@helse-forde.no

'Department of Surgery, Førde Central Hospital, Svanehaugvegen 1, 6812

Førde, Norway

Full list of author information is available at the end of the article
}

C The Author(s). 2020 Open Access This article is distributed under the terms of the Creative Commons Attribution 4.0 International License (http://creativecommons.org/licenses/by/4.0/), which permits unrestricted use, distribution, and reproduction in any medium, provided you give appropriate credit to the original author(s) and the source, provide a link to the Creative Commons license, and indicate if changes were made. The Creative Commons Public Domain Dedication waiver (http://creativecommons.org/publicdomain/zero/1.0/) applies to the data made available in this article, unless otherwise stated. 


\section{Background}

Living with an ostomy defined as an opening constructed to extract bodily waste (flatus, faeces, or urine) can be challenging both physically and psychosocially. Patients with an ostomy lack a functional sphincter, and the waste empties involuntary in a pouch [1]. The importance of follow-up with a stoma care nurse (SCN) has been widely discussed, and novel approaches to tailor care more closely to the needs of these patients, particularly those struggling with ostomy-related adaptation and with a reduced health-related quality of life (HRQoL) could be useful. We here define ostomy-related adaptation as an individual's degree of adaptive or maladaptive adjustment to specific important ostomy issues [2], while we define HRQoL as a multidimensional construct of the individual's perception of physical, psychological, and social dimensions of health in a more broader and generic sense [3]. Ostomy care is in this project defined as the SCN's follow-up of the patient in an outpatient clinic setting.

Although the major indications for an intestinal or urinary ostomy include malignancy in the urinary or gastrointestinal tract and indications for intestinal ostomies may include inflammatory bowel diseases, a wide range of diagnoses can lead to the need for an ostomy. Regardless of the need for ostomy surgery, the literature consistently describes negative feelings such as fear, insecurity, denial, shame, pain, dissatisfaction with appearance, and anger among patients after ostomy surgery [4]. These feelings could persist and result in a feeling of social stigma. Ostomy can involve changes in daily life, such as skin care, nutrition, clothing, work, leisure, social activities, sleeping, sexuality, and physical activity [4-8]. Two studies on short and long term adjustment found lowered adjustment in certain areas, such as sport and physical exercises, work, sexuality, body image, embarrassing accidents (such as leakage), and they would have done more things if they didn't have had an ostomy [9, 10].

The HRQoL of ostomy patients is often negatively affected [11, 12], and may be lower than that in the general populations [13-17]; in fact, the HRQoL may remain low over several years after surgery [18]. The negative predictors for the adaptation process and HRQoL include a dependence on others for the management of the ostomy, leakage or fear of leakage, odour, noise and equipment failure, complications, and lack of social support and education [5, 18-20].

Lopez and Descerado et al. identified that dialog between the patient and the healthcare team was a major tool for a better adaptation to life with an ostomy [21]. Several studies specify that education programs, social support, and follow-up before and after surgery are imperative factors [7, 20, 22-26]. However, studies describing novel methods for consultations between the SCN and patients, and the manner in which patients provide feedback are lacking. There seems to be a gap in the literature when it comes to longitudinal studies describing how the patients adapt to life with ostomy.

A promising and relatively new clinical approach is the use of routine outcome monitoring (ROM) of the patient's progress in treatment over time, using questionnaires that could include a clinical feedback system (CFS) in the consultation [27]. This approach has been successful in improving practice and outcomes within physical and mental-health care in a range of patient groups [28]. ROM/CFS can provide the clinician with detailed information that may have been overlooked or not previously regarded as important [29]. This gives clinicians' better information on the state of the patient's progress, and the clinician can share and discuss these results with the patient. Moreover, this knowledge can be used to evaluate, and if necessary, adjust treatment [30]. ROM/CFS is also valuable because it seems to have a positive effect on the communication and therapeutic relationship between the patient and the clinician $[28,31]$. Web-based ROM/CFS appear to be a particularly feasible methodology in the healthcare setting, where it can be used to both monitor and evaluate treatment and as a research tool [32]. In the present paper, we describe a new model of patient reported outcomes (PRO) and CFS in ostomy care and a plan for evaluation.

\section{Aims}

1. To study experiences and satisfaction with care in patients using PRO/CFS during the first 12 months after having an ostomy.

2. To study changes in the Ostomy Adjustment Scale (OAS) - profile and HRQoL in patients using PRO/ CFS during the first 12 months after having an ostomy.

3. To study which items in the OAS score the patients using PRO/CFS rate as the most challenging during the first 12 months after having an ostomy.

\section{Methods}

In this longitudinal study, we will include patients who have undergone urostomy, colostomy, or ileostomy operations, and who are included in the routine follow-up program of the outpatient ostomy clinic at the Department of Surgery, Førde Central Hospital from April 2018 to June 2021. The potential number of eligible new patients having ostomy pr. year is approximately 35 . In this study, the ostomy patients 
undergo follow-up consultations at 3, 6, and 12 months after surgery. The inclusion criteria are (a) > 18 years of age; (b) living with a colostomy, ileostomy, or urostomy for up to 12 months; and (c) being able to talk, read and write Norwegian.

\section{Ethics and safety}

The study conforms to the principles outlined in the Declaration of Helsinki. The Regional Committee of Ethics in Medicine, West-Norway, has approved the study protocols (registration numbers: 2016/255). Checkware, an agency which is used by the Western Norway Health Hospital Trust, has delivered the electronic version of the questionnaires. The study will use the highest security level possible in Norway to protect patient information. Each study participant will use his/her BankID with a code device or a cell phone, and a personal password. Questionnaires that are answered in the paper form, as well as the patient consent form, will be stored in a safe place in the Research Department.

\section{Power calculations}

We base our power calculations on analysis of change in OAS over time. A priori power calculation should as be based on the smallest clinical meaningful difference over time. No minimally important effect size at a group level have been defined for OAS. Thus, we have to rely on research and consensus regarding this issue for PRO measures sin general. An effect size of 0.5 is a conservative estimate of clinical significance, and that effect sizes down to 0.3 may also be meaningful [33]. Consequently, our power calculation is based on a two-sided paired test (effect size $=0.4$, correlation between measures of $0.3,90 \%$ power, $p \leq$ 0.05 ) indicating that at least 68 paired observations would be required to detect reasonably robust $95 \% \mathrm{CI}$ estimates of changes in the OAS. In order to take into account attrition we will aim to include $100 \mathrm{pa}-$ tients in the study.

\section{Intervention}

SCNs educated in accordance with the requirements of the World Council of Enterostomal Therapists (WCET) conducts the systematic follow-up of ostomy patients in the outpatient clinic of Førde Central Hospital. The follow-up by SCN includes information, education in ostomy-related topics, skin and ostomy inspection and treatment, and to optimize the ostomy equipment as recommended in international and national guidelines and standards [34-36]. See Appendix 1 which describes the content in the previous and new follow-up consultations.
In the literature, $\mathrm{SCN}$ is also known as Wound, Ostomy and Continence Nurse or Enterostomal Therapist, but have been consistently regarded as $\mathrm{SCN}$ in this article.

The intervention in this study involves a ROM/CFS program where ostomy patients complete questionnaires before and after their consultation in the outpatient ostomy clinic approximately 3, 6, and 12 months after the surgery. The patient is expected to have the same SCN in all planned consultations.

\section{Procedure}

When patients are invited to the 3-month follow-up visit in the outpatient department, they receive written information about the study and a participation consent form. Patients who want to participate in the study provide consent to their SCN. The SCN adds the questionnaires into the electronic database, and makes the questionnaires available to the patient.

On the day of the 3-month follow-up session, the participants can answer the questionnaires via a computer/Ipad at home before they arrive for the consultation or they can sit in a designated area in the waiting room for approximately $20 \mathrm{~min}$ prior to the consultation. During these $20 \mathrm{~min}$, the patient can $\log$ onto the data system using his/her electronic identification to answer the questionnaires about sociodemographic data and clinical data, and complete the OAS and Short Form 36 (SF-36) questionnaires.

For patients without any personal electronic identification (BankID using a code device or a cell phone), the SCN allows the patients to access the questionnaires using a one-time code. In special cases, if the SCN logs on using his/her own code, the SCN will remain in the same room as the patient when he/she answers the questions for security reasons. Alternatively, the SCN asks the questions during the consultation and enter the answers into the database with the assistance of the patient, or we permit the patient to fill in a paper version of the questionnaire prior to admission for the consultation, and the SCN enters the answers into the database. In cases where the patient answers the questions via a computer or Ipad, the answers are available to the SCN before the patient arrives for the consultation.

During the consultation, the patient and SCN discuss the results and agree on the issues that may contribute to better adaptation and management of life with an ostomy. For patients who need help to answer the forms, the SCN discusses the answers with the patient.

The OAS items with the lowest scores will be presented first in the electronic report Low scores (1-3 on a Likert scale from 1 to 6) indicate that the patient is struggling with adaptation to the ostomy in 
the relevant life area, for example in work, leisure activities, travelling or body image and sexuality. The patient and SCN can then discuss strategies to achieve better adaptation. Similarly, the eight SF-36 domain scores are shown as bar charts, with different colours for each consultation. The patient and SCN can discuss methods for achieving adaptation in the items with low scores. For example, if the patient scores low on items relating to how safe he/she feels with regard to the ostomy bag, the SCN can discuss the type of bag used, the procedure, skin inspection and how to manage a situation with leakage from the equipment. Items that are scored better (4-6 on a Likert scale from 1 to 6) indicate better adaptation to the ostomy, and the SCN may choose not to focus on those during the discussion. Low SF-36 scores indicate reduced HRQoL, and the patient and SCN can discuss the reason for the low score and identify strategies to achieve a higher HRQoL.

The clinical part of the consultation, such as changing of the pouch, and observation of the ostomy and skin, is conducted before or after the discussion, depending on the preferences of the patient.

At the end of the consultation, the patient and SCN summarise the agreements and select the date and time for the next appointment.

After the consultation, the patients completes the Generic Short Patient Experiences Questionnaire scale (GS-PEQ) related to their experience during the consultation. This scale will be answered in a paper version.

The SCN fills in a set of clinical data after each consultation. The form has 2 parts, including a part about the diagnosis, type of ostomy, duration of ostomy, stoma site marking, treatment, and information given before surgery, and the patient's degree of management of ostomy practical skills when he/she left the hospital after surgery. The SCN records these data after the first consultation. The second part of the form includes weight, description of the peristomal skin and the ostomy, complications, characteristics of body waste, any new diagnoses, and treatments initiated after surgery. The second part of the form is recorded after all consultations. This clinical data is also used in the discussion during the consultation and the findings will be available for future consultations.

\section{Implementation of the intervention}

A pilot study was started with 15 patients, to evaluate, and if necessary, to adjust the intervention or other practical aspects of the study. In particular, we needed to test the functioning of the technology, and create a seamless process from the patient's response to the graphical presentation of the questionnaire metrics during the consultation. As there was no need for technical or practical changes to the study plan based on that study, the patients in the pilot study are included in the actual study.

Two SCNs, including one of the study authors (KLI), will conduct the systematic follow-up of the patients. During the project, we will register the number of patients carrying their Bank ID, the number of patients requiring help from an SCN to fill in the scales, and the number of patients who needed to fill in a paper version of the questionnaires.

The project will be implemented within the usual follow-up schedule for the ostomy patients, and the findings will be documented in the hospital's patient administrative system, as done previously. The project will not burden the patients in terms of money or a significant amount of additional time.

\section{Outcomes}

\section{GS-PEQ}

To evaluate the patient's experiences and satisfaction with ROM/CFS we will use the GS-PEQ. The Norwegian knowledge centre for health services questionnaires has developed and validated the 12 -item scale, which contains questions about patient satisfaction and experiences with somatic outpatient services in Norway [37] (See Appendix 2).

\section{OAS}

To focus on the adaptation process, we will use a questionnaire without detailed evaluations of the ostomy equipment or clinical complications regarding the ostomy and skin, but with a focus on the consequences of body change and the adaptation to the new condition. Therefore, the Norwegian version of the OAS, originally developed by Olbrisch in 1983, will be used as measure of the primary outcome [2] This 34-item scale developed by a psychiatrist, ostomy care nurses, patients, and students records a patient's subjective adaptation to the physical, psychological, and social changes that occur after ostomy surgery. In particular, it records the patient's employment status, marital relationship, social functioning status, selfimage, and social life. The scale contains questions about the patient's care of their ostomy, their opinions about the instructions they received about their ostomy, and their feelings about the SCN as well as the surgeon responsible for their ostomy surgery [2]. All the items are scored on a Likert scale from 1 (strongly agree) to 6 (strongly disagree); the total OAS scores can vary from 34 to 204 , with a higher score indicating good adaptation to the ostomy [2]. The reliability of the OAS, as measured by Cronbach's alpha, has been reported to be 0.87 [2], 0.89 [38], and 0.93 [9] in previous reports, and its testretest correlation coefficients have been reported to 
be 0.72 [2] and 0.69 [9]. Previous studies also support the instrument's construct validity [9, 39]. Mary Ellen Olbrisch, the researcher who designed the instrument, provided permission to use this scale for clinical research in Norway. In the electronic version, a graph shows the OAS sum scores from current and previous consultations. Furthermore, the patient's most challenging metrics are reported first in the electronic report (see Appendix 2).

\section{SF-36}

The SF-36 is a well-validated, generic health scale that measures the outcomes that are known to be the most directly affected by disease and treatment [40]. The SF36 is a self-reported questionnaire with eight subscales that measure physical functioning, body pain, role limitations due to physical health problems, role limitations due to emotional or personal problems, emotional wellbeing, social functioning, energy/fatigue, and perceived general health. Each subscale has a total score; the instrument can also be divided into two summary scores (based on factor analysis using oblique rotation): physical component scores (PCS; including domains such as physical function, physical role function, pain, and general health), and mental health component scores (MCS; including domains such as emotional role limitations, vitality, social function, and mental health). A single item that provides an indication of the perceived change in health is also included. The scores in each domain are converted to a scale from 0 to 100; higher scores are associated with better health-related quality of life (HRQoL). The scale has shown robust validity and reliability [41] (See Appendix 2).

\section{Statistical analysis}

To assess the frequencies of the sociodemographic and clinical variables we will use descriptive statistics. Missing data on the questionnaires will be handled according to the procedures described for each questionnaire [2, 42]. In the analysis of patient experiences and satisfaction with care, descriptive results (number and percent) on each item of GS-PEQ at the 1 year follow up will be presented (aim 1). To study changes in OAS, PCS and MCS we will use longitudinal regression models with time as a (categorical) explanatory variable with an unstructured correlation matrix (aim 2). These models use data from all patients, even in patients with partially missing data. To study which items in the OAS score the patients range as the most challenging during first 12 months postoperative, mean item scores will be calculated and ranked (aim 3). SPSS software (version 25; IBM, Armonk, NY) will be used for all analyses.

\section{User involvement}

A user panel including two patients with an ostomy has been involved in designing the study. Patients have coproduced the selection of questionnaires, and the workflow of the ROM/CFS according to patient's needs. The user panel will also help interpreting findings in order to better disseminate aspects that are important from the patient's point of view.

\section{Discussion}

This study could be useful for improving the practice of follow-up by SCN for ostomy patients, as patients gives a more detailed description of his/her degree of adaptation to life with an ostomy. Furthermore, this may lead to more individualised consultations based on patients' needs.

A strength of the current study is that the ROM/CFS concept has been implemented in other patient groups at Førde Health Trust. Thus, we have in-house resources available to implement and support this project both technically and clinically. The GS-PEQ scale is developed in Norway for use in evaluation of outpatient somatic healthcare [37]. The OAS, is well validated and in use worldwide [2, 38, 39, 43], and has been validated for use in the Norwegian population $[9,13]$.. The SF-36 questionnaire, is also widely used and well validated $[41,44,45]$. The sociodemographic and clinical forms are developed in cooperation with a reference group, including ostomy patients and SCNs with considerable experience in outpatient clinics. Feedback from both patients and SCNs not in the project can offer new perspectives and provide valuable feedback regarding the item's relevance and ease of answering the questions.

This present study also has certain challenges. Based on our experience, we find that not all patients bring Bank ID equipment with them for the consultation, and may hence lack the necessary identification code to access the questionnaire. Therefore, we need to focus on minimising this occurrence during the project. Another challenge is the familiarity of patients in the use of IPad or computers, and their comfort with answering many questions (96 items) The lack of qualitative evaluation of the implementation is a methodological limitation. Our hospital has a small ostomy outpatient clinic with two SCNs (including the author), which may serve as a strength and limitation. For example, the close relationship between the author and the clinic can help with implementation, as the author can closely monitor the clinical part of the project; however, this close relationship also emphasises the need for a qualitative evaluation by external researchers on the new method of consultation. At present, the project does not have the financial resources to conduct qualitative studies, although this will be reconsidered if funding is secured. 


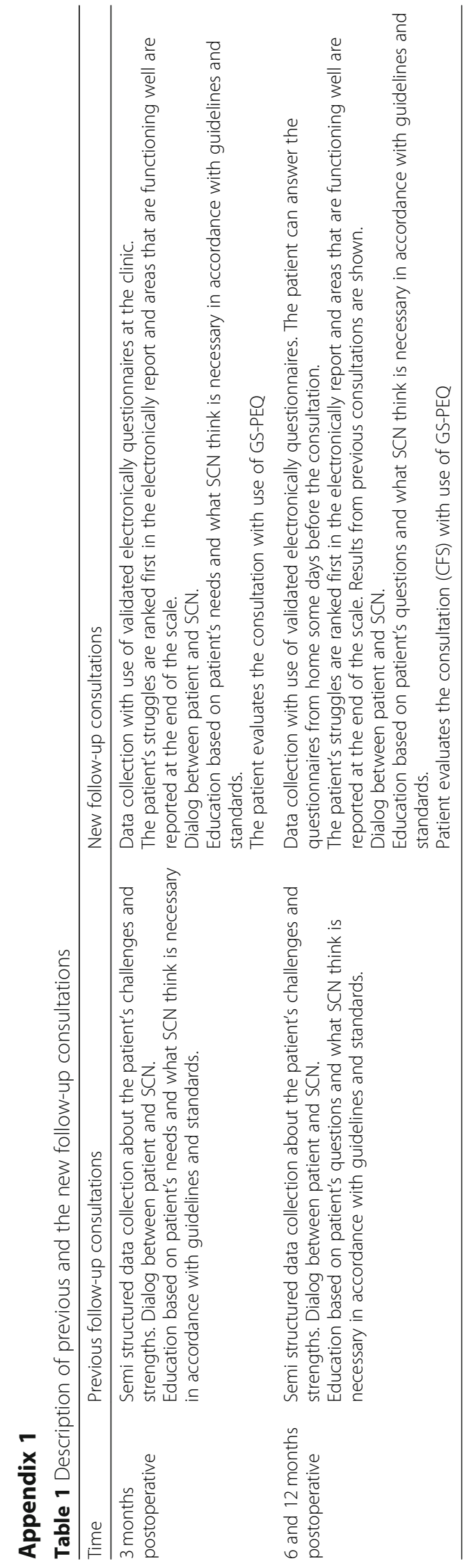




\section{Appendix 2}

\section{Descriptions and links to the main questionnaires in English}

\section{The Generic Short Patient Experiences Questionnaire (GS-PEQ). HS-PEQ contains 12} questions about patient satisfaction and experiences with somatic outpatient services in Norway. Link to free full-text of the items: https:// bmchealthservres.biomedcentral.com/articles/10.11 86/1472-6963-11-88

2. Ostomy Adjustment Scale (OAS). OAS has 34 items on adjustment to life with an Ostomy. Link to free full-text of the items: https://www.owm.com/article/cross-sectional-study-determinewhether-adjustment-ostomy-can-predict-health-related-andor Note: To see the items go to the results section of the paper, and click Table 2.

3. Short-Form-36: The SF-36 is a self-reported questionnaire with eight subscales that measure physical functioning, body pain, role limitations due to physical health problems, role limitations due to emotional or personal problems, emotional well-being, social functioning, energy/fatigue, and perceived general health.

Link to free full text of the questionnaire: https:// www.rand.org/health-care/surveys_tools/mos/36item-short-form/survey-instrument.html

\section{Abbreviations}

CFS: Clinical Feedback System; GS-PEQ: Generic Short Patient Experiences Questionnaire; HRQoL: Health related quality of life; IC: Ileal conduit; OAS: Ostomy Adjustment Scale; ROM: Routine Outcome Monitoring: SCN: Stoma Care Nurse; SF-36: Short form 36

\section{Acknowledgements}

We thank the patients in the user panel for all help during the design of the study.

\section{Authors' contributions}

$\mathrm{KLI}$ and JRA planned the study protocol and KLI critically reviewed the literature, and drafted and edited all versions of the manuscript. TEO made important suggestions in the development of the clinical forms. All authors made suggestions that improved the design and all authors approved the final manuscript.

\section{Funding}

The study is funded by Norwegian Nursing Organisation to cover the salary of the PhD student for 4 years (75\% payment each year). The research group "Folkehelse, Livsstil og Overvekt" of the Research Department, Førde Health Trust, covers the operating costs for the study for 4 years.

\section{Availability of data and materials}

The dataset generated during this study will not be publicly available as the patient consent and approval from the Regional Committee for Medical and Health Research Ethics prevents sharing of individual patient level data in public repositories. However, the data will be available from the corresponding author upon reasonable request.

\section{Ethics approval and consent to participate}

This investigation conforms to the principles outlined in the Declaration of Helsinki. The Regional Committee of Ethics in Medicine, West-Norway, approves the study protocol (registration number: 2016/255). Informed consent to participate in the study is obtained from all individual participants included in the study.

\section{Consent for publication}

Informed consent was obtained from all individual study participants to publish data collected from the participants.

\section{Competing interests}

The authors declare that they have no competing interests.

\section{Author details}

'Department of Surgery, Førde Central Hospital, Svanehaugvegen 1, 6812 Førde, Norway. ${ }^{2}$ Western Norway University of applied Sciences, Førde, Norway. ${ }^{3}$ Department of Surgery, Haukeland University Hospital, Bergen, Norway. ${ }^{4}$ Centre of Health Research, Førde Hospital Trust, Førde, Norway.

Received: 23 February 2019 Accepted: 18 December 2019

Published online: 15 January 2020

\section{References}

1. Kiliç E, Taycan O, Bellı AK. The effect of permanent Ostomy on body image, self-esteem, marital adjustment, and sexual functioning. Turk J Psychiatry. 2007;18(4):1-8

2. Olbrisch ME, Jansen MA. Development and validation of the Ostomy adjustment scale. Rehabil Psychol. 1983;28:3-12.

3. Cramer JA, Spilker B. Quality of life and pharmacoeconomics: an introduction. Philadelphia: Lippincott Williams \& Wilkins, Lippincott-Raven Publishers; 1998

4. Lopes A, Decesaro M. The Adjustments Experienced by Persons With an Ostomy: An Integrative Review of the Literature. King of Prussia: HMP Communications. 2014;60:34

5. Villa G, Manara DF, Brancato T, Rocco G, Stievano A, Vellone E, Alvaro R. Life with a urostomy: a phenomenological study. Appl Nurs Res. 2018:39:46-52.

6. Haughey S, McGroggan G. Living well with a stoma: a descriptive evaluation. Gastrointest Nurs. 2017:15:41-8.

7. Lim SH, Chan SWC, He H-G. Patients' experiences of performing self-care of stomas in the initial postoperative period. Cancer Nurs. 2015:38:185-93.

8. Claessens I, Probert R, Tielemans C, Steen A, Nilsson C, Andersen BD, Størling ZM. The Ostomy life study: the everyday challenges faced by people living with a stoma in a snapshot. Gastrointest Nurs. 2015;13:18-25.

9. Indrebø LK, Andersen RJ, Natvig KG. The Ostomy adjustment scale: translation into Norwegian language with validation and reliability testing. J Wound Ostomy Continence Nurs. 2014;41:357-64.

10. Fingren J, Lindholm E, Petersen C, Hallen A-M, EJOwm C. A Prospective, Explorative Study to Assess Adjustment 1 Year After Ostomy Surgery Among Swedish Patients. 2018;64:12.

11. Vonk-Klaassen SM, de Vocht HM, den Ouden MEM, Eddes EH, Schuurmans MJ. Ostomy-related problems and their impact on quality of life of colorectal cancer ostomates: a systematic review. Qual Life Res. 2016;25: 125-33.

12. Brown H, Randle J. Living with a stoma: a review of the literature. J Clin Nurs. 2005:14:74-81.

13. Indrebø KL, Natvig GK, Andersen JR. A cross-sectional study to determine whether adjustment to an Ostomy can predict health-related and/or overall quality of life. Ostomy Wound Manage. 2016;62:50.

14. Furukawa C, Sasaki Y, Matsui K, Morioka I. Health-related quality of life and its relevant factors in Japanese patients with a urostomy. J Wound Ostomy Continence Nurs. 2013;40:165-70.

15. Nichols RT. Quality of life in US residents with Ostomies as assessed using the SF36v2. J Wound Ostomy Continence Nurs. 2015;42:71-8. 
16. Näsvall P, Dahlstrand U, Löwenmark T, Rutegård J, Gunnarsson U, Strigård K. Quality of life in patients with a permanent stoma after rectal cancer surgery. Qual Life Res. 2017;26:55-64.

17. Boraii S. A descriptive study to assess quality of life in Egyptian patients with a stoma. Ostomy Wound Manage. 2017;63:28-33.

18. Cerruto MA, D'Elia C, Cacciamani G, De Marchi D, Siracusano S, lafrate M, Niero M, Lonardi C, Bassi P, Belgrano E. Behavioural profile and human adaptation of survivors after radical cystectomy and ileal conduit. Health Qual Life Outcomes. 2014;12:46.

19. Recalla S, English K, Nazarali R, Mayo S, Miller D, Gray M. Ostomy care and management: a systematic review. J Wound Ostomy Continence Nurs. 2013; 40:489-500.

20. Merandy K. Factors related to adaptation to cystectomy with urinary diversion: an integrative review. J Wound Ostomy Continence Nurs. 2016;43: 499-508.

21. Lopes A, Decesaro M. Adjustments experience by persons with an ostomy: a integrative review of the literature. Ostomy Wound Manage [Internet] 2014;60(10):34-42.

22. Faury S, Koleck M, Foucaud J, M'bailara K, Quintard B. Patient education interventions for colorectal cancer patients with stoma: a systematic review. Patient Educ Couns. 2017;100:1807-19.

23. Johnson T. Follow-up care of stoma patients: a systematic literature review. Gastrointest Nurs. 2012;10:30-6.

24. Beaver K, Latif S, Williamson S, Procter D, Sheridan J, Heath J, Susnerwala S, Luker K. An exploratory study of the follow-up care needs of patients treated for colorectal cancer. J Clin Nurs. 2010;19:3291-300.

25. Millan M, Tegido M, Biondo S, García-Granero E. Preoperative stoma siting and education by stomatherapists of colorectal cancer patients: a descriptive study in twelve Spanish colorectal surgical units. Color Dis. 2010;12:e88-92.

26. Zhang YJ-E, Wong YFK, You YL-M, Zheng YM-C, Li YQ, Zhang YB-Y, Huang YM-R, Ye YX-M, Liang YM-J, Liu YJ-L. Effects of Enterostomal nurse telephone follow-up on postoperative adjustment of discharged colostomy patients. Cancer Nurs. 2013;36:419-28.

27. Riemer M, Rosof-Williams J, Bickman L. Theories related to changing clinician practice. Child and Adolescent Psychiatric Clinics. 2005;14:241-54.

28. Kd J. A chance for change: building an outcome monitoring feedback system for outpatient mental health care. Leiden: Clinical, Health and Neuropsychology, Faculty of Social and Behavioural Sciences, Leiden University; 2012.

29. Bickman L, Kelley SD, Breda C, de Andrade AR, Riemer M. Effects of routine feedback to clinicians on mental health outcomes of youths: results of a randomized trial. Psychiatr Serv. 2011;62:1423-9.

30. Lambert MJ. Prevention of treatment failure: the use of measuring, monitoring, and feedback in clinical practice. Washington DC: American Psychological Association; 2010.

31. Carlier IVE, Meuldijk D, Van Vliet IM, Van Fenema E, Van Der Wee NJA, Zitman FG. Routine outcome monitoring and feedback on physical or mental health status: evidence and theory. J Eval Clin Pract. 2012;18:104-10.

32. de Beurs E, Den Hollander-Gijsman M, van Rood Y, van Der Wee N, Giltay E, van Noorden M, van Der Lem R, van Fenema E, Zitman F. Routine outcome monitoring in the Netherlands: practical experiences with a web-based strategy for the assessment of treatment outcome in clinical practice. Clin Psychol Psychother. 2011;18:1-12.

33. Sloan JA, Cella D, Hays RD. Clinical significance of patient-reported questionnaire data: another step toward consensus. J Clin Epidemiol. 2005; 58(12):1217-9.

34. Zulkowski K, Ayello E, Stelton S. WCET international Ostomy Guideline. Perth: WECT; 2014.

35. Society Clinical Guideline WOCN. Management of the Adult Patient With a Fecal or Urinary Ostomy-An Executive Summary. J Wound Ostomy Continence Nurs. 2018;45:50.

36. WCET International Ostomy Guideline.

37. Sjetne IS, Bjertnaes OA, Olsen RV, Iversen HH, GJBhsr B. The Generic Short Patient Experiences Questionnaire (GS-PEQ): identification of core items from a survey in Norway, vol. 11; 2011. p. 88

38. Burckhardt CS, Eisenberg MG. The Ostomy adjustment scale: further evidence of reliability and validity. Rehabil Psychol. 1990;35:149-55.

39. Brydolf M, Berndtsson I, Lindholm E, Berglund B. Evaluation of a Swedish version of the Ostomy adjustment scale. Scand J Caring Sci. 1994:8:179-83.

40. Ware JE, Sherbourne CD. The MOS 36-item short-form health survey (SF-36): I. Conceptual Framework and Item Selection. Med Care. 1992;30:473-83.
41. Hays RD, Morales LS. The RAND-36 measure of health-related quality of life. Ann Med. 2001;33:350-7.

42. Ware J, Kosinski M, Gandek B. SF-36 health survey: manual and interpretation guide Lincoln. Rl: QualityMetric Incorporated; 2000.

43. J-e Z, Wong FKY, Zheng M-C, Hu A-I, H-q Z. Psychometric evaluation of the ostomy adjustment scale in Chinese cancer patients with colostomies. Cancer Nurs. 2015;38:395-405.

44. Aaronson NK, Muller M, Cohen PD, Essink-Bot M-L, Fekkes M, Sanderman R, Sprangers MA, Te Velde A, Verrips E. Translation, validation, and norming of the Dutch language version of the SF-36 health survey in community and chronic disease populations. J Clin Epidemiol. 1998;51:1055-68.

45. Loge JH, Kaasa S, Hjermstad MJ, Kvien TK. Translation and performance of the Norwegian SF-36 health survey in patients with rheumatoid arthritis. I. Data quality, scaling assumptions, reliability, and construct validity. J Clin Epidemiol. 1998;51:1069-76.

\section{Publisher's Note}

Springer Nature remains neutral with regard to jurisdictional claims in published maps and institutional affiliations.

Ready to submit your research? Choose BMC and benefit from

- fast, convenient online submission

- thorough peer review by experienced researchers in your field

- rapid publication on acceptance

- support for research data, including large and complex data types

- gold Open Access which fosters wider collaboration and increased citations

- maximum visibility for your research: over $100 \mathrm{M}$ website views per year

At BMC, research is always in progress.

Learn more biomedcentral.com/submissions 